\title{
The $(\mathbf{n}, \gamma)$ cross sections of short-living $s$-process branching points
}

\author{
K. Sonnabend*, A. Mengoni ${ }^{\dagger}$, P. Mohr*, T. Rauscher**, K. Vogt* and A. \\ Zilges* \\ *Institut für Kernphysik, Technische Universität Darmstadt, Germany \\ ${ }^{\dagger}$ CERN, Geneva, Switzerland \\ ${ }^{* *}$ Institut für Physik, Universität Basel, Switzerland
}

\begin{abstract}
An experimental method to determine the $(\mathrm{n}, \gamma)$ cross section of short-living $s$-process branching points using data of the inverse $(\gamma, \mathrm{n})$ reaction is presented. The method was used to observe the branching point nucleus ${ }^{95} \mathrm{Zr}$ because the elemental abundance patterns corresponding to this branching point cannot be reproduced by full stellar models and a possible error source is the neutron capture cross section of ${ }^{95} \mathrm{Zr}$. The analysis of the experiment is still under progress, we will outline the current status in this manuscript.
\end{abstract}

\section{INTRODUCTION}

The nucleosynthesis of the elements heavier than iron is today mainly explained by three processes: $s-, r$-, and $p$-process. $s$ - and $r$-process are based on neutron-capture reactions with adjacent $\beta$-decays while the $p$-process is governed by photodisintegration reactions such as $(\gamma, \mathrm{n}),(\gamma, \mathrm{p})$, or $(\gamma, \alpha)$.

The distinction between $s$ - and $r$-process is due to the different neutron densities $n_{\mathrm{n}}$ involved. During $s$-process nucleosynthesis $n_{\mathrm{n}}$ is in the order of $10^{8}$ neutrons $/ \mathrm{cm}^{3}$ while typical $r$-process sites deal with $n_{\mathrm{n}}>10^{20}$ neutrons $/ \mathrm{cm}^{3}[1]$. Hence, the neutron capture rates $\lambda_{(\mathrm{n}, \gamma)}$ are larger than typical $\beta$-decay rates $\lambda_{\beta}$ during $r$-process nucleosynthesis $\left(\lambda_{(\mathrm{n}, \gamma)} \gg \lambda_{\beta}, r\right.$ : rapid). In the $s$-process the situation is the other way round $\left(\lambda_{(\mathrm{n}, \gamma)} \ll \lambda_{\beta}\right.$, $s$ : slow) and the involved nuclei are close to the valley of $\beta$-stability.

However even in as $s$-prozess scenario, if the half-life $T_{1 / 2}$ is long enough and the neutron capture cross section high enough another neutron capture might take place and the path "branches" out. Therefore, these nuclides are called branching points of the $s$ process. The branching ratio - i.e. how often each of the paths is taken - determines the corresponding elemental abundance patterns. Using a model for $s$-process nucleosynthesis and knowing precisely the nuclear physics input (half-life $T_{1 / 2}$ and Maxwellian averaged capture cross section (MACS) at typical $s$-process temperatures $k T=30 \mathrm{keV}$ ) it is possible to determine the astrophysical parameters temperature $T$ and neutron density $n_{\mathrm{n}}$.

In the so-called classical approach temperature $T$ and neutron density $n_{\mathrm{n}}$ are considered to be constant. Thus, three different components produced with different astrophysical parameters are needed to reproduce the observed abundances of $s$-only nuclei: the weak component corresponds to mass numbers $A<90$ while the strong component only 
describes the termination of the $s$-process path at lead and bismuth. In between these borders the isotopes belong to the so-called main $s$-process [2]. The abundances of $s$ only isotopes that are not affected by a branching can be reproduced with a mean square deviation of about $3 \%$ using this simple model [3].

A more realistic approach is a full stellar model, e.g. the AGB star model described in Ref. [4]. These models need very precise nuclear physics input data to reproduce the $s$-only abundances. Especially the MACS of several branching points hamper the reliability of the predictions: due to the lack of experimental data the theoretical predictions of the MACS sometimes show a broad spread (see [5]).

A direct measurement of the MACS of the branching points is only possible if their half-lives are in the order of years (e.g. ${ }^{147} \mathrm{Pm}$ with $T_{1 / 2}=2.62 \mathrm{yr}$ [6]). In the case of short-living branching points with half-lives of about a dozen days or even less, adequate samples are not available. Thus, direct measurements are possible.

To solve this problem we have investigated an experimental method using the data of the inverse $(\gamma, \mathrm{n})$ reaction as described in Section 2 . Section 3 presents ${ }^{95} \mathrm{Zr}$ focusing on astrophysical aspects as well as on the constraints of our experimental method for this nuclide. The present status of the analysis is summarized in Section 3.3 In Section 4 we describe the next step in the data evaluation.

\section{EXPERIMENTAL METHOD}

The aim of our experimental method is to constrain the theoretical predictions of the MACS of short-living $s$-process branching nuclei. Short-living under $s$-process conditions denotes half-lives of about a dozen days or less, i.e. direct measurements are not possible due to the lack of adequate samples. Therefore, our method is based on data of the inverse $(\gamma, n)$ reaction.

The first step is to measure the so-called energy-integrated cross section $I_{\sigma}$ using the photoactivation technique:

$$
I_{\sigma}=\int_{S_{\mathrm{n}}}^{E_{\max }} \sigma(E) \cdot N_{\gamma}\left(E, E_{\max }\right) \cdot \mathrm{d} E
$$

with $\sigma(E)$ being the $(\gamma, \mathrm{n})$ cross section and $N_{\gamma}\left(E, E_{\max }\right)$ the experimental photon flux.

We use the monoenergetic electron beam provided by the S-DALINAC [7] to produce a continuous bremsstrahlung spectrum by fully stopping the beam in a thick rotating copper target. The spectrum ranges from $E=0$ to $E_{\mathrm{e}^{-}}$, so that $E_{\max }$ of Eq. (11) equals $E_{\mathrm{e}}$. Due to the location of our setup directly behind the injector of the S-DALINAC, our maximum energy is limited to about $E_{\mathrm{e}^{-}}=10 \mathrm{MeV}$ presently [8]. While the shape of $N_{\gamma}\left(E, E_{\max }\right)$ is well known the absolute value has to be determined by a relative measurement with gold as a calibration standard.

After irradiating the targets the yield $Y$ of the activation is measured by counting the $\gamma$-rays emitted in the decay of the produced radioactive nuclei with a HPGe-detector that is well-shielded against background by several layers of lead. The yield $Y$ is proportional to the energy-integrated cross section $I_{\sigma}$ with the constant of proportionality being explained in detail in Ref. [9]. 
Because the $(\gamma, \mathrm{n})$ cross section of ${ }^{197} \mathrm{Au}$ is known to behave like

$$
\sigma_{\mathrm{Au}}(E)=146.2 \mathrm{mb} \cdot\left(\frac{E-S_{\mathrm{n}}}{S_{\mathrm{n}}}\right)^{0.545}
$$

near threshold [10], a predicted energy dependence of the target's cross section $\sigma_{\text {target }}(E)$ can be normalized to the observed ratio $I_{\sigma}^{\text {target }} / I_{\sigma}^{\text {Au }}$ by a correction factor $f$ for each measured energy $E_{\max }$. In doing so, either a parametrization or a theoretical calculation of $\sigma_{\text {target }}(E)$ can be used. If $f$ depends on $E_{\max }$ the shape of $\sigma_{\text {target }}(E)$ is not accurately described (compare Eq. 1) and $f$ can not be used for the further procedure without modifications.

Although the photoactivation technique is very sensitive, several limitations concerning the activation reaction ${ }^{A} \mathrm{X}(\gamma, \mathrm{n})^{A-1} \mathrm{X}$ and the following decay of ${ }^{A-1} \mathrm{X}$ have to be taken into account. To test the energy dependece of the cross section $\sigma_{\text {target }}(E)$ it is necessary to measure at different energies $E_{\max }$. Because our present setup is limited to 10 $\mathrm{MeV}$, isotopes with neutron thresholds $S_{\mathrm{n}}>9 \mathrm{MeV}$ cannot be checked.

If one wants to use naturally composed targets, the fraction of the isotope of interest must not be too small and the activation of other isotopes should not produce too much background radiation when the decay of ${ }^{A-1} \mathrm{X}$ is observed. To reach a good peak-tobackground ratio in the measured spectra only a few $\gamma$-rays with high branchings should be emitted in the decay of ${ }^{A-1} \mathrm{X}$. Furthermore, the analyzed $\gamma$-decays should not be part of a multi-level decay and their energies $E_{\gamma}$ should not allow self-absorption in the target to avoid additional uncertainties in the analysis.

If the normalization of a theoretical prediction of $\sigma_{\text {target }}(E)$ yields a constant factor $f$, we use $f$ to correct the MACS predicted in the same model with equal parameters. This second step of using the correction factor for the inverse reaction is based on the detailed balance assumption. In our case, scaling the MACS with a constant factor $f$ equals scaling the $\gamma$-ray strength function. For further discussion see [11].

Although the corrected MACS is still based on a theoretical prediction, its value is constrained by experimental knowledge of the inverse reaction due to the determination of $f$. Thus, it is more reliable than a value purely based on theory.

\section{THE BRANCHING POINT ${ }^{95} \mathrm{ZR}$}

\subsection{Position on the $s$-process path}

The branching point ${ }^{95} \mathrm{Zr}$ is located near the borderline between the weak and the main component of the $s$-process [2]. Therefore, the prediction of the elemental abundance patterns corresponding to this branching is a crucial test for the validity of a full stellar model described in [4].

Several problems concering the prediction of the zirconium abundance patterns measured in SiC grains are reported in Ref. [12]. The uncertainty in the predicted MACS of ${ }^{95} \mathrm{Zr}$ is mentioned as one of the possible error sources due to the wide range of predicted values from $23 \mathrm{mb}$ to $126 \mathrm{mb}$ at $k T=30 \mathrm{keV}$ (see [13], [14], [15], and [16]). Another 


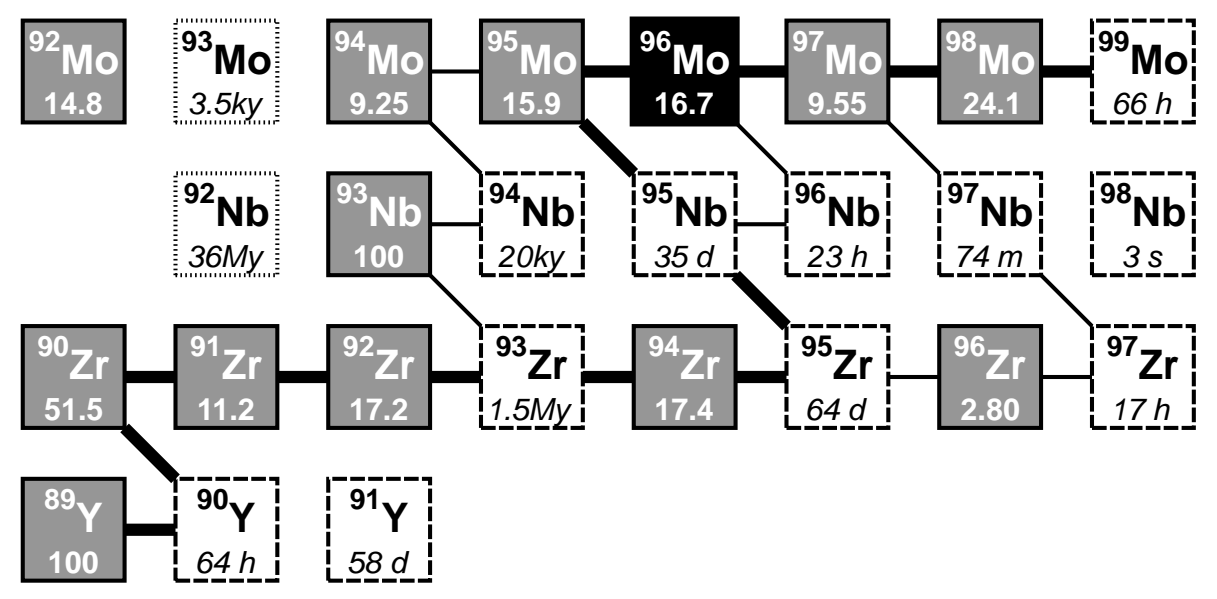

FIGURE 1. The $s$-process path around the branching point ${ }^{95} \mathrm{Zr}$. The main flow of the $s$-process is marked with thick lines, thinner lines indicate branches. Grey shaded boxes illustrate stable isotopes with the numbers at the bottom of the boxes being their natural abundances. The black box of ${ }^{96} \mathrm{Mo}$ stresses that we have an $s$-only nucleus. The white boxes display $\beta$-instable nuclei: dashed boundaries are used for $\beta^{-}$-decay, dotted lines are used to indicate $\beta^{+}$- or $\varepsilon$-instable isotopes. In this cases the numbers at the bottom of the boxes are the half-lives of the ground-states.

reason for the deviation between the measured and predicted abundance patterns might be the uncertainty in the half-life $T_{1 / 2}$ of ${ }^{95} \mathrm{Zr}$ at $s$-process temperatures. However, as confirmed by a recent measurement [17] the first excited level is at $E=954 \mathrm{keV}$ and thus, is not significantly populated at $k T=30 \mathrm{keV}$. Hence, the half-life of ${ }^{95} \mathrm{Zr}$ does not depend on temperature under $s$-process conditions and can be omitted as an error source because of its small error: $T_{1 / 2}\left({ }^{95} \mathrm{Zr}\right)=(64.032 \pm 0.006) \mathrm{d}[18]$.

\subsection{Constraints of the experiment}

To use our experimental method the constraints described in Section 2 have to be fullfilled for the activation reaction ${ }^{96} \mathrm{Zr}(\gamma, \mathrm{n}){ }^{95} \mathrm{Zr}$. The threshold of this reaction is $S_{\mathrm{n}}=7854 \mathrm{keV}$, which can be easily reached at our setup providing energies up to 10 $\mathrm{MeV}$. The abundance of ${ }^{96} \mathrm{Zr}$ in a naturally composed target is $2.8 \%$ and thus, high enough for the activation method.

The properties of the decay of ${ }^{95} \mathrm{Zr}$ are summarized in Fig. 2 The characteristics claimed in Section 2 are achieved for $E_{\gamma}=724.2 \mathrm{keV}$ and $E_{\gamma}=756.7 \mathrm{keV}$.

Due to the half-life of ${ }^{95} \mathrm{Zr}$ of about 64 days it is mandatory to observe the decay for several hours to reach good statistics. During this time the $\beta^{-}$-decay of the daughter nucleus ${ }^{95} \mathrm{Nb}$ also starts to take place. The assignment of the measured $\gamma$-rays to the decays of ${ }^{95} \mathrm{Zr}$ or ${ }^{95} \mathrm{Nb}$ can either be done by a determination of the observed half-life or by comparing their different behaviour in time. As shown in Fig. 3 the ratio of the two peaks at 724.2 and $756.7 \mathrm{keV}$ remains unchanged if the measurement takes place different times after activation. Thus, both lines correspond to the same decay and are 


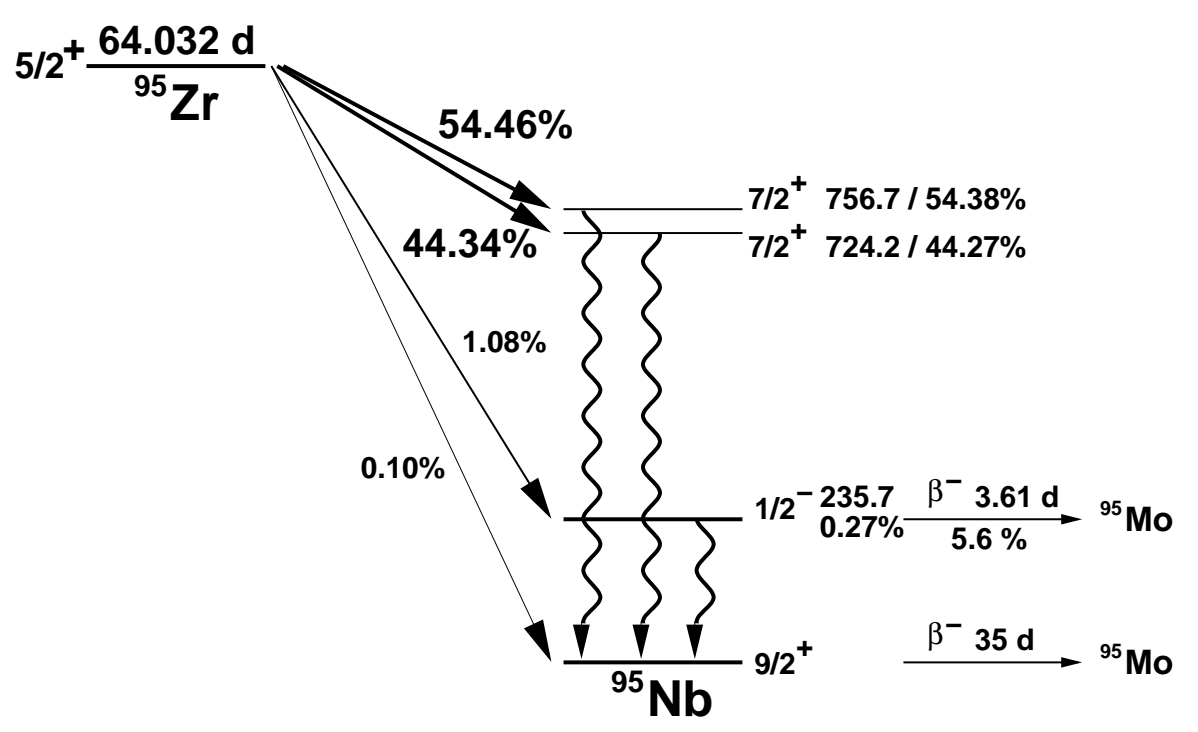

FIGURE 2. Section of the decay scheme of ${ }^{95} \mathrm{Zr}$ based on [18]

identified to be the most prominent lines of the decay of ${ }^{95} \mathrm{Zr}$. In contrast, the peak at $765.8 \mathrm{keV}$ becomes more and more dominant with time. This behaviour is consistent with the one expected of a line corresponding to the decay of the daughter nucleus ${ }^{95} \mathrm{Nb}$. Actually, the energy $E_{\gamma}=765.8 \mathrm{keV}$ is exactly the energy supposed for the most

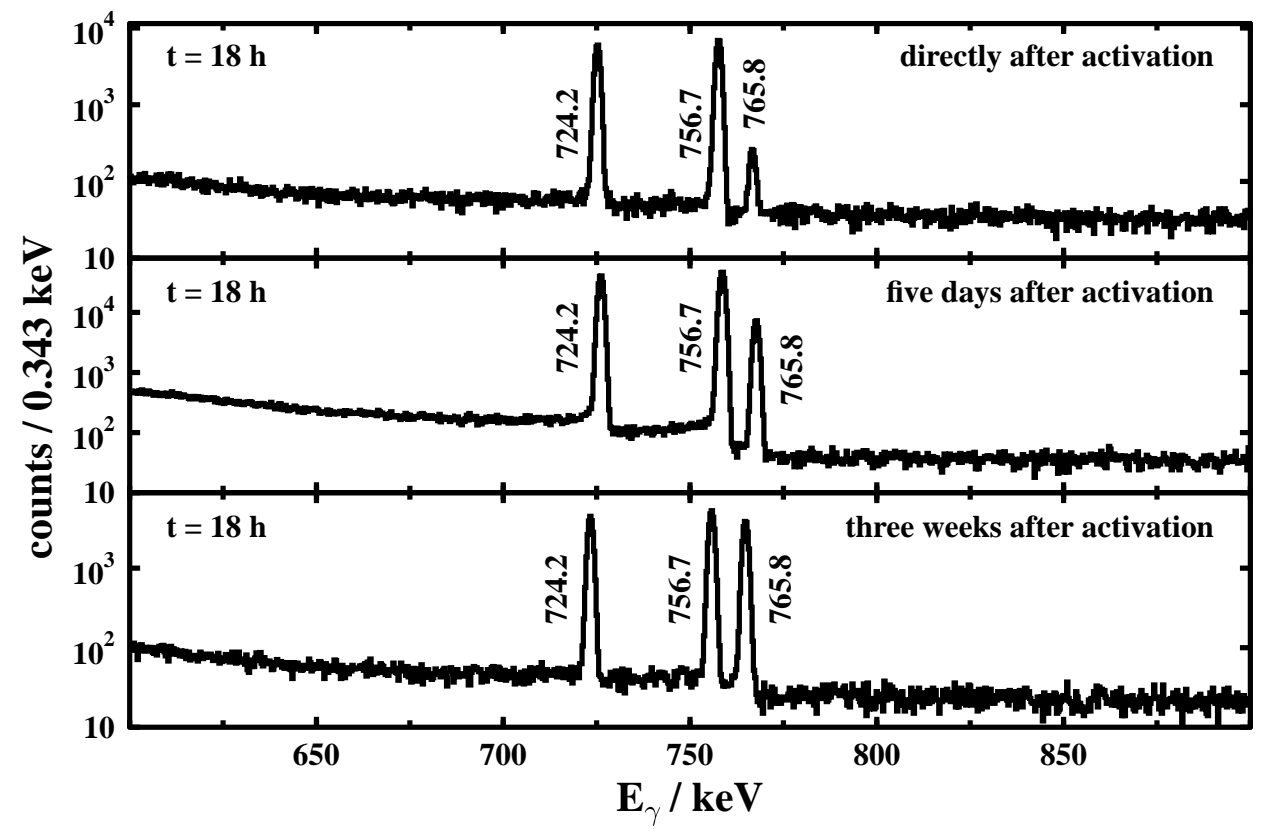

FIGURE 3. Typical decay spectra of ${ }^{95} \mathrm{Zr}$ each measured for $t=18 \mathrm{~h}$. From the top to the bottom panel the time between activation and start of the measurement is changing as indicated on the top right. For further explanation see text. 
prominent line of this decay.

\subsection{Current status of analysis}

The photoactivation of zirconium has been done at six different energies $E_{\text {max }}$ ranging from 8325 to $9900 \mathrm{keV}$. The lower limit is due to the separation energy of the used calibration standard: $S_{\mathrm{n}}(\mathrm{Au})=8071 \mathrm{keV}$. In a first approach, we used a parametrization that describes the energy dependence of the $(\gamma, n)$ cross section near threshold:

$$
\sigma(E)=\sigma_{0} \cdot\left(\frac{E-S_{\mathrm{n}}}{S_{\mathrm{n}}}\right)^{p}
$$

In this case, the exponent $p$ changes the shape of the energy dependence, whereas the factor $\sigma_{0}$ is responsible for the absolute value of $\sigma(E)$. Therefore, $\sigma_{0}$ corresponds to the correction factor $f$ of Section 2, Due to the fact that this parametrization is derived from the time reversal symmetry of $(n, \gamma)$ and $(\gamma, n)$ reactions, the exponent $p$ is connected to the wave-character of the emitted neutron: an exponent of $p=0.5+l$ corresponds to an $l$-wave decay. Thus, exponents less than 0.5 cannot be explained in this basic approach.

However, if a least-mean-square-fit of our data to this parametrization is carried out using $\sigma_{0}$ and $p$ as free parameters, we obtain $p=0.339$ and $\sigma_{0}=31.81 \mathrm{mb}$ as shown in Fig. 4 The single results $\sigma_{0}\left(E_{\max }\right)$ fluctuate around the mean value, thus, the overall energy dependence of $\sigma(E)$ seems to be described accurately.

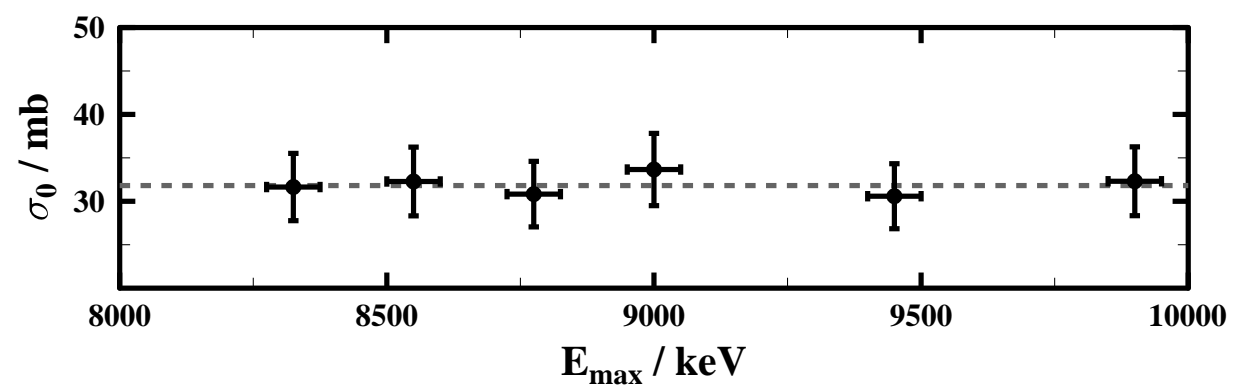

FIGURE 4. Result of a least-mean-square-fit of Eq. (3) to the measured data at different energies $E_{\max }$. The dots are the results for $\sigma_{0}$ derived with an exponent of $p=0.339$ while the dashed line is the mean value $\sigma_{0}=31.81 \mathrm{mb}$. The horizontal error bars are due to uncertainties in the beam energy.

The result $p<0.5$ can be explained by assuming a resonance on top of the smooth behaviour described by Eq. (3). We measure the energy integrated cross section $I_{\sigma}$ and compare it with the result of Eq. (1) using $\sigma(E)$ of Eq. (3). Thus, we average the measured $(\gamma, \mathrm{n})$ cross section $\sigma(E)$ over the energy interval ranging from $S_{\mathrm{n}}$ to $E_{\max }$. A resonance in this energy region would yield the measured bigger $I_{\sigma}$.

The smaller $p$, the steeper is the rise of the cross section at the threshold energy $S_{\mathrm{n}}$. Hence, the calculated $I_{\sigma}$ becomes bigger and suits the measured value. Thus, we accept the derived values for $p$ and $\sigma_{0}$ as a description of the mean energy dependence of the $(\gamma, \mathrm{n})$ cross section: $\sigma(E)=31.81 \mathrm{mb} \cdot\left(\left(E-S_{\mathrm{n}}\right) / S_{\mathrm{n}}\right)^{0.339}$.

Fig. [5] shows the mean $\sigma(E)$ and a theoretical prediction of the $(\gamma, n)$ cross section $\sigma_{\text {theo }}(E)$ based on Hauser-Feshbach calculations [19, 20].If the shapes of the two energy 


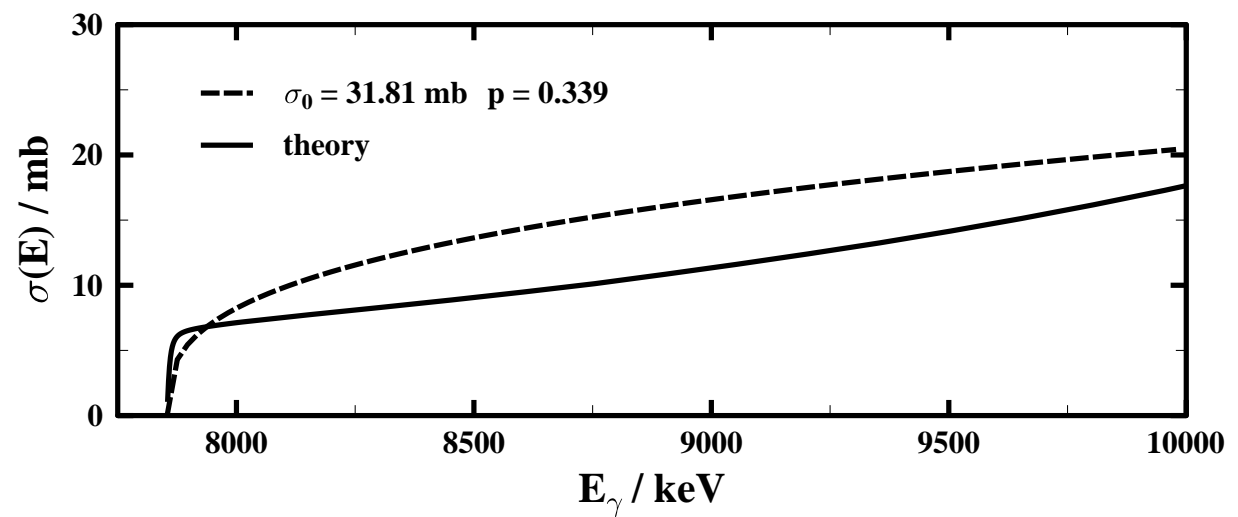

FIGURE 5. Comparison of the energy dependence of the cross section $\sigma(E)$ for the reaction ${ }^{96} \mathrm{Zr}(\gamma, \mathrm{n})$. The solid line is a theoretical prediction based on Hauser-Feshbach calculations. The dashed line is the result of a least-mean-square-fit of the parametrization described in Eq. (3) to our data.

dependences are compared, a significant deviation is obvious: the theoretical prediction rises very steeply close to the threshold, but its slope becomes flatter $50 \mathrm{keV}$ above threshold. In contrast, our parametrization is not so steep near threshold but more continuously rising.

Although the parametrization of $\sigma(E)$ describes only a mean energy dependence of the cross section, the different behaviour illustrates that the energy dependence of the theoretical prediction does not suit the data as well. Obviously, no resonances are predicted by theory. A correction factor $f$, derived as explained in Section 2 is only a mean correction. $f$ can compensate the absence of resonances in theory. Furthermore, $f$ is a function of $E_{\max }$. Therefore, the MACS of ${ }^{95} \mathrm{Zr}$ predicted by this theory cannot simply be corrected by this mean value of $f$ but the (n, $\gamma)$ cross section must be corrected by $f\left(E_{\gamma}\right)$ before calculating the MACS. $f\left(E_{\gamma}\right)$ can be calculated weighting $f\left(E_{\max }\right)$ by $\sigma(E) \cdot N_{\gamma}\left(E, E_{\max }\right)$. For this purpose, it is necessary to measure the $(\gamma, \mathrm{n})$ cross section as close as possible to the reaction threshold to locate the position of the resonances.

So far, the experiment was limited by the threshold of the calibration standard. The lowest measured energy was $E_{\max }=8325 \mathrm{keV}$ i.e. still about $500 \mathrm{keV}$ above the threshold $S_{\mathrm{n}}$ of ${ }^{96} \mathrm{Zr}$. In order to measure as close as possible near the target's threshold the neutron separation energy of a new calibration standard has to be lower.

In addition to a low neutron separation energy the calibration standard has to fullfill other characteristics: despite the general features mentioned in Section 2, the half-life $T_{1 / 2}$ of the produced radioactive isotope has to be short so that the counting can be done in short times with good statistics.

We decided to use ${ }^{187} \mathrm{Re}$ with a neutron separation energy of only $S_{\mathrm{n}}=7359 \mathrm{keV}$ and a natural abundance of $62.6 \%$. The half-life of the produced ${ }^{186} \mathrm{Re}$ is $T_{1 / 2}=3.72 \mathrm{~d}$ and allows short measuring times although the branching of the two dominant $\gamma$-decays is quite low: $E_{\gamma}=137.2 \mathrm{keV}$ with $9.42 \%$ and $E_{\gamma}=122.6 \mathrm{keV}$ with $0.6 \%$. Self-absorption in the rhenium target limits the amount of material, however, based on experience of former experiments [11] very thin rhenium targets with a thickness of $d=50 \mu \mathrm{m}$ should provide enough activity after a typical activation. 


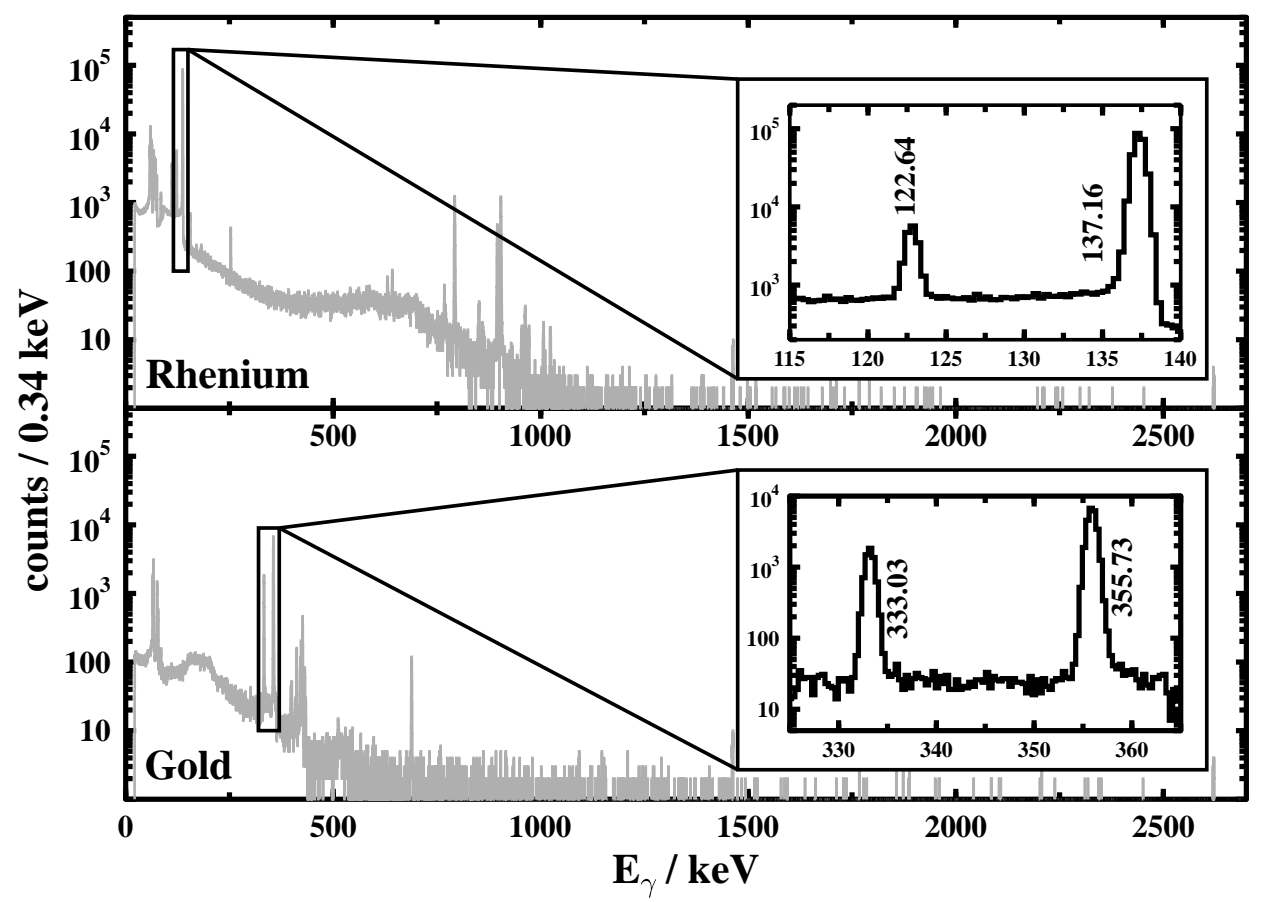

FIGURE 6. Comparison of the decay spectra of the calibration standards gold and rhenium. The inlays show the $\gamma$ peaks that are used for the calibration. The peak-to-background ratio is in the same order of magnitude for both standards.

${ }^{187} \mathrm{Re}$ was measured in May 2003 and the analysis is still under progress. Fig. 6 compares the spectra of rhenium and gold measured after an activation at $E_{\max }=$ $8325 \mathrm{keV}$. The upper panel shows the spectrum of rhenium measured for three hours directly after the activation. The peaks between 750 and $1000 \mathrm{keV}$ correspond to the decay of ${ }^{184} \operatorname{Re}$ that is produced by ${ }^{185} \operatorname{Re}(\gamma, \mathrm{n})$. Due to these lines the two peaks of the decay of ${ }^{186} \mathrm{Re}$ at $E_{\gamma}=137.2 \mathrm{keV}$ and $E_{\gamma}=122.6 \mathrm{keV}$ are located above a high background. However, as shown in the inlay the peak-to-background ratio of both lines is good enough to analyze the peak volumina with a low statistical error. For comparison the inlay in the lower panel shows the two most prominent lines of a gold spectrum also measured for three hours. The peak-to-background ratio is in the same order of magnitude.

\section{SUMMARY AND OUTLOOK}

The precise knowledge of the (n, $\gamma$ ) cross section of the $s$-process branching point ${ }^{95} \mathrm{Zr}$ is a crucial input parameter to test the reliability of the AGB star model. Due to the very wide range of the theoretically predicted values a measurement observing the inverse reaction with the photoactivation technique was carried out at the S-DALINAC in June 2002.

During the analysis of the data several problems occured: The energy dependence 
of the $(\gamma, \mathrm{n})$ cross section of ${ }^{96} \mathrm{Zr}$ extracted from the data differs significantly from the theoretical prediction. In our opinion, the deviation is due to resonances that are not included in the used theory. Therefore, the experimental method described in Section 2 determines an energy dependent correction factor $f\left(E_{\gamma}\right)$ that cannot be used to correct the predicted MACS of ${ }^{95} \mathrm{Zr}$ without further studies.

Hence, the experiment was extended in May 2003 by measuring the $(\gamma, \mathrm{n})$ cross section of ${ }^{96} \mathrm{Zr}$ closer to the reaction threshold. Therefore, a new calibration standard with lower neutron separation energy was measured during the same beam-time. The analysis of the new standard ${ }^{187} \mathrm{Re}$ is still under progress, preliminary results like the spectrum shown in Fig. 6 are encouraging that it will work as well as our first standard ${ }^{197} \mathrm{Au}$.

Once a description of the $(\gamma, n)$ cross section of ${ }^{187} \operatorname{Re}$ near threshold is available the new data point of ${ }^{96} \mathrm{Zr}$ taken at $E_{\max }=8100 \mathrm{keV}$ has to be added to the existing analysis. Therewith, the discrepancy between the experimental result and the theoretical prediction will hopefully disappear or at least become explainable. The outcome of the whole analysis that is the experimentally confirmed MACS of ${ }^{95} \mathrm{Zr}$ can then be used in the AGB star model and might solve the problems in explaining the observed zirconium abundance patterns in $\mathrm{SiC}$ grains.

In future, we will take more data points at different energies $E_{\max }$ in order to reduce difficulties due to resonances on top of the cross section. If the distance between two data points $\Delta E_{\max }$ is smaller, the determination of the location of a resonance is possible with higher precision.

\section{ACKNOWLEDGMENTS}

The authors would like to thank R. Gallino (Torino, Italy) and F. Käppeler (Karlsruhe, Germany) for fruitful discussions concerning $s$-process modelling. This work was supported by the Deutsche Forschungsgemeinschaft (contracts Zi 510/2-2 and SFB 634) and Swiss NSF (grants 2124-055832.98, 2000-061822.00, 2024-067428.01).

\section{REFERENCES}

1. Beer, H., Encyc. Astron. Astroph. 2, 1866 (2000).

2. Käppeler, F., Prog. Part. Nucl. Phys. 43, 419 (1999).

3. Käppeler, F., Gallino, R., Busso, M., Picchio, G., and Raiteri, C., Astrophys. J. 354, 630 (1990).

4. Lugaro, M., Herwig, F., Lattanzio, J., Gallino, R., and Straniero, O., Astrophys. J. 586, 1305 (2003).

5. Bao, Z. Y., Beer, H., Käppeler, F., Voss, F., Wisshak, K., and Rauscher, T., At. Data Nucl. Data Tables 76, 70 (2000).

6. Reifarth, R., Arlandini, C., Heil, M., Käppeler, F., Sedyshev, P., Mengoni, A., Herman, M., Rauscher, T., and Gallino, R., Astrophys. J. 582, 1251 (2003).

7. Richter, A., Prog. Part. Nucl. Phys. 44, 3 (2000).

8. Mohr, P., Enders, J., Hartmann, T., Kaiser, H., Schiesser, D., Schmitt, S., Volz, S., Wissel, F., and Zilges, A., Nucl. Instr. and Meth. A 423, 480 (1999).

9. Vogt, K., Mohr, P., Babilon, M., Enders, J., Hartmann, T., Hutter, C., Rauscher, T., Volz, S., and Zilges, A., Phys. Rev. C 63, 055802 (2001).

10. Vogt, K., Mohr, P., Babilon, M., Bayer, W., Galaviz, D., Hartmann, T., Hutter, C., Rauscher, T., Sonnabend, K., Volz, S., and Zilges, A., Nucl. Phys. A707, 241 (2002). 
11. Sonnabend, K., Mohr, P., Vogt, K., Zilges, . A., Mengoni, A., Rauscher, T., Beer, H., Käppeler, F., and Gallino, R., Astrophys. J. 583, 506 (2003).

12. Lugaro, M., Davis, A., Gallino, R., Pellin, M., Straniero, O., and Käppeler, F., Astrophys. J. 593, 486 (2003).

13. Holmes, J., Woosley, S., Fowler, W., and Zimmermann, B., At. Data Nucl. Data Tables 18, 305 (1976).

14. Toukan, K., and Käppeler, F., Astrophys. J. 348, 357 (1990).

15. Rauscher, T., and Thielemann, F.-K., At. Data Nucl. Data Tables 75, 1 (2000).

16. Goriely, S., Nuclear astrophysics data base, http://www.astro.ulb.ac.be/Nucdata/ (2003).

17. Sonnabend, K., Mohr, P., Zilges, A., Hertenberger, R., Wirth, H.-F., Graw, G., and Faestermann, T., Phys. Rev. C 68, 048802 (2003).

18. NNDC Online Data Service, ENSDF database, http://www.nndc.bnl.gov/nndc/ensdf/ (2003).

19. Wolfenstein, L., Phys. Rev. 82, 690 (1951).

20. Hauser, W., and Feshbach, H., Phys. Rev. 87, 366 (1952). 